\title{
ON THE LEFSCHETZ NUMBER FOR ITERATES OF CONTINUOUS MAPPINGS ${ }^{1}$
}

\author{
H. O. PEITGEN
}

\begin{abstract}
We give an elementary proof of the $(\bmod p)$-congruence between the Lefschetz numbers of a continuous mapping and its $p$ th iterate, $p$ prime, and deduce some results about periodic mappings.
\end{abstract}

The following proposition can be obtained in a less general situation from theorems of Zabreiko and Krasnosel'skiī [10] and Steinlein [9] by using the normalization property of the fixed point index. However, the proofs in [9] and [10] are analytical and quite difficult. Moreover, the normalization property requires some deeper considerations (cf. Dold [1]). In this paper we present a short and appropriate proof using only basic algebraic and topological arguments.

Proposition. Let $X$ be a compact Hausdorff space such that $\breve{H}^{*}(X ; \mathbf{Z})$ is finitely generated. Then $\Lambda(f) \equiv \Lambda\left(f^{p}\right) \bmod p$, whenever $f$ is a continuous map on $X$ and $p$ is prime.

For the proof of the proposition we need the following basic

Lemma. Let $A$ be a matrix of integers and let $p$ be prime. Then trace $A$ $\equiv \operatorname{trace} A^{p} \bmod p$.

Proof. If $A$ is in $\mathbf{Z}^{n^{2}}$, we denote by $\Pi: \mathbf{Z}^{n^{2}} \rightarrow \mathbf{Z}_{p}^{n^{2}}$ and by $\pi: \mathbf{Z} \rightarrow \mathbf{Z}_{p}$ the corresponding projections. We have $\Pi\left(A^{p}\right)=(\Pi(A))^{p}$ and $\pi(\operatorname{trace} A)$ $=$ trace $\Pi(A)$. Let $\lambda_{1}, \ldots, \lambda_{n}$ be the eigenvalues of $\Pi(A)$. Since the $\lambda_{i}$ are roots of a polynomial with coefficients in $\mathbf{Z}_{p}$, then $\sum_{i=1}^{n} \lambda_{i}$ is defined in the extension field $\mathbf{Z}_{p}\left(\lambda_{1}, \ldots, \lambda_{n}\right)$ of $\mathbf{Z}_{p}$. Notice that $\mathbf{Z}_{p}\left(\lambda_{1}, \ldots, \lambda_{n}\right)$ is a field of characteristic $p$, hence $\left(\sum_{i=1}^{n} \lambda_{i}\right)^{p}=\sum_{i=1}^{n} \lambda_{i}^{p}$. Since trace $\Pi(A)=\sum \lambda_{i}$ and trace $(\Pi(A))^{p}=\sum \lambda_{i}^{p}$ are in $\mathbf{Z}_{p}$, we have $\sum \lambda_{i}=\left(\sum \lambda_{i}\right)^{p}=\sum \lambda_{i}^{p}$ in $\mathbf{Z}_{p}$. Thus we get

$$
\pi(\operatorname{trace} A)=\operatorname{trace} \Pi(A)=\operatorname{trace}(\Pi(A))^{p}=\pi\left(\operatorname{trace} A^{p}\right) .
$$

Proof of Proposition. From Spanier [8, p. 335], we have that the following diagram with exact rows commutes:

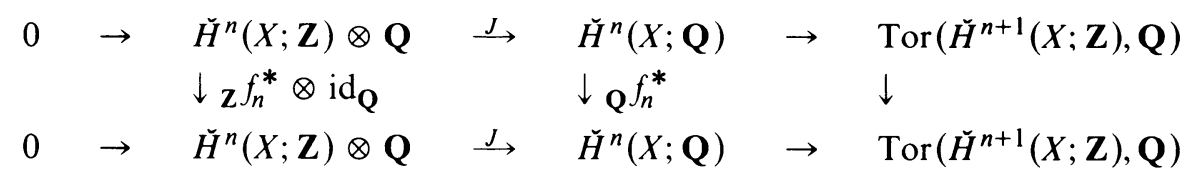

Received by the editors August 13, 1974.

AMS (MOS) subject classifications (1970). Primary 55C20; Secondary 55C25, 55C35.

Key words and phrases. Lefschetz number, iterates, fixed point index, periodic mappings.

1 The contents of this paper were presented at the meeting on Problems in Nonlinear Functional Analysis held in Bonn, July 22-26, 1974. 
Since $\operatorname{Tor}(A, \mathbf{Q})=0$ for any abelian group $A$, we have that $J$ is an isomorphism and, therefore,

$$
\operatorname{trace}\left({ }_{\mathbf{Z}} f_{n}^{*} \otimes \mathrm{id}_{\mathbf{Q}}\right)=\operatorname{trace}\left(J^{-1} \circ \mathbf{Q} f_{n}^{*} \circ J\right)=\operatorname{trace}\left({ }_{\mathbf{Q}} f_{n}^{*}\right) .
$$

Let $F$ be a free subgroup and $T$ the torsion subgroup of $\check{H}^{n}(X ; \mathbf{Z})$ such that $\breve{H}^{n}(X ; \mathbf{Z})=F \oplus T$, and let $e_{1}, \ldots, e_{r}$ be a basis for $F$. Then a basis for $\breve{H}^{n}(X ; \mathbf{Z}) \otimes \mathbf{Q}$ is given by $e_{1} \otimes 1, \ldots, e_{r} \otimes 1$. If we choose $a_{i j}$ in $\mathbf{Z}$ and $t$ in $T$ such that $\mathbf{Z} f_{n}^{*}\left(e_{i}\right)=\sum_{j=1}^{r} a_{i j} e_{j}+t$, then we have

$$
\mathbf{z} f_{n}^{*} \otimes \mathrm{id}_{\mathbf{Q}}\left(e_{i} \otimes 1\right)=\sum_{j=1}^{r} a_{i j} e_{j} \otimes 1 .
$$

Hence ${ }_{\mathbf{Z}} f_{n}^{*} \otimes \mathrm{id}_{\mathbf{Q}}$ is given by a matrix of integers. Now

$$
\Lambda(f)=\sum_{n}(-1)^{n} \text { trace } \mathbf{Q} f_{n}^{*}=\sum_{n}(-1)^{n} \text { trace }_{\mathbf{Z}} f_{n}^{*} \otimes \mathrm{id}_{\mathbf{Q}} .
$$

From the Lemma we have

$$
\operatorname{trace}\left({ }_{\mathbf{Z}} f_{n}^{*} \otimes \mathrm{id}_{\mathbf{Q}}\right) \equiv \operatorname{trace}\left({ }_{\mathbf{Z}} f_{n}^{*} \otimes \mathrm{id}_{\mathbf{Q}}\right)^{p} \bmod p .
$$

Finally,

$$
\begin{aligned}
\operatorname{trace}\left(\mathbf{z} f_{n}^{*} \otimes \mathrm{id}_{\mathbf{Q}}\right)^{p} & =\operatorname{trace}\left(J^{-1} \circ \mathbf{Q} f_{n}^{*} \circ J\right)^{p} \\
& =\operatorname{trace}\left(J^{-1} \circ\left(\mathbf{Q} f_{n}^{*}\right)^{p} \circ J\right)=\operatorname{trace}\left(\mathbf{Q} f_{n}^{*}\right)^{p}
\end{aligned}
$$

which proves the proposition.

It can be easily seen that the same proof works in the case of multivalued mappings in the sense of Powers [7].

Our interest in the proposition arose from the attempt of proving the (mod p)-theorem of Zabreiko and Krasnosel'skii [10] and Steinlein [9] by purely topological means and from its usefulness in asymptotic fixed point theory (cf. Fenske and Peitgen [2] and Peitgen [6]).

We close with a few observations and remarks.

(1) The following is a generalization of results due to Floyd [3], [4] and Halpern [5]. The proofs in [3] and [4] use the Smith special homology groups, and the proof in [5] is based on the Kelley-Spanier characteristic.

THEOREM. Let $X$ be a compact Lefschetz space such that $\breve{H}^{*}(X ; \mathbf{Z})$ is finitely generated and let $T$ be a fixed point free periodic transformation of period $s=p^{r}$, $p$ prime, operating on $X$. Then $p$ divides the Euler characteristic $\chi(X)$.

Proof. From the proposition we have that

$$
\chi(X)=\Lambda\left(T^{p^{r}}\right) \equiv \Lambda\left(T^{p^{r-1}}\right) \equiv \cdots \equiv \Lambda\left(T^{p}\right) \equiv \Lambda(T)=0
$$

where all congruences are $\bmod p$.

We add in passing that the same argument yields the divisibility result in the case when $T$ is a fixed point free transformation on $X$ and $\left.\mathbf{Q}^{\left(T^{s}\right.}\right)^{*}=\mathrm{id}$. 
Corollary . Let $X$ be a compact Lefschetz space such that $\check{H}^{*}(X ; \mathbf{Z})$ is finitely generated, and let $T$ be a periodic transformation of period $s=p^{r}$, p prime, operating on $X$. Assume that $\chi(X) \not \equiv 0 \bmod p$; then $T$ has a fixed point.

(2) Set $\lambda_{p}=p^{-1}\left(\Lambda\left(g^{p}\right)-\Lambda(g)\right)$. The proposition tells us that $\lambda_{p}$ is an integer invariant for $g$. It seems to be of some interest to determine $\lambda_{p}$ especially in view of the following observations. Set

$$
\chi_{p}(X)=\sum_{n}(-1)^{n} \operatorname{dim}_{\mathbf{Z}_{p}} \check{H}^{n}\left(X ; \mathbf{Z}_{p}\right),
$$

whenever this number is defined. Assume that $X$ is a finite-dimensional compact Hausdorff space such that $\check{H}^{*}(X ; \mathbf{Z})$ is finitely generated. Notice that a standard argument using Theorem 10 in Spanier [8, p. 335], yields $\chi(X)$ $=\chi_{p}(X)$. Consider the very special case when $g$ is a fixed point free transformation of prime period $p$ operating on $X$. Then we have that $\lambda_{p}=\chi_{p}(X / G)$. Here $G$ is the group generated by $g$ and $X / G$ is the orbit space. This follows from Floyd [3], [4]. Furthermore, combining another result of Floyd [3] and the proposition, we have that

$$
\Lambda(g) \equiv \Lambda\left(g^{p}\right)=\chi(X) \equiv \chi_{p}\left(X^{G}\right) \bmod p
$$

when $g$ is not necessarily fixed point free.

We would like to remark that a purely topological approach for a proof of the $(\bmod p)$-theorem for the fixed point index is the following.

Let $f: U \rightarrow \mathbf{R}^{n}$ be continuous, where $U$ is open in $\mathbf{R}^{n}$. Let $p$ be prime and denote by $F$ (resp. $F_{p}$ ) the fixed point set of $f$ (resp. $f^{p}$ ) in $U$. Assume that $F$ and $F_{p}$ are compact and that $f^{p}$ has no fixed points on the boundary of $U$ and $f\left(F_{p}\right) \subset U$. Then the Krasnosel'skiī-Zabreiko-Steinlein theorem is equivalent to the commutativity of the following diagram:

$$
\begin{aligned}
H_{n}\left(S^{n} ; \mathbf{Z}_{p}\right) \rightarrow H_{n}\left(S^{n}, S^{n}-F ; \mathbf{Z}_{p}\right) \rightarrow H_{n}\left(U, U-F ; \mathbf{Z}_{p}\right) & \stackrel{(i-f)^{*}}{\longrightarrow} H_{n}\left(\mathbf{R}^{n}, \mathbf{R}^{n}-\{0\} ; \mathbf{Z}_{p}\right) \\
H_{n}\left(S^{n} ; \mathbf{Z}_{p}\right) \rightarrow H_{n}\left(S^{n}, S^{n}-F_{p} ; \mathbf{Z}_{p}\right) \rightarrow H_{n}\left(U, U-F_{p} ; \mathbf{Z}_{p}\right) \mid & \stackrel{\left(i-f^{p}\right)^{*}}{\longrightarrow} H_{n}\left(\mathbf{R}^{n}, \mathbf{R}^{n}-\{0\} ; \mathbf{Z}_{p}\right)
\end{aligned}
$$

Using coefficients in $\mathbf{Z}$ instead of $\mathbf{Z}_{p}$, the rows define a fixed point index for $f$ (resp. $f^{p}$ ) (cf. Dold [1]). It is immediate from Dold's paper that it suffices to prove commutativity of the right rectangle where $F$ is replaced by $F_{p}$.

\section{REFERENCES}

1. A. Dold, Fixed point index and fixed point theorems for Euclidean neighborhood retracts, Topology 4 (1965), 1-8. MR 33 \# 1850.

2. C. Fenske and H. O. Peitgen, Repulsive fixed points of multivalued transformations and the fixed point index, Math. Ann. (to appear). 
3. E. E. Floyd, On periodic maps and the Euler characteristics of associated spaces, Trans. Amer. Math. Soc. 72 (1952), 138-147. MR 13, 673.

4. - Periodic maps via Smith theory, Seminar on Transformation Groups, Ann. of Math. Studies, no. 46, Princeton Univ. Press, Princeton, N.J., 1960, Chap. III.

5. B. R. Halpern, Fixed points for iterates, Pacific J. Math. 25 (1968), 255-275. MR 41 \# 1039.

6. H. O. Peitgen, Asymptotic fixed point theorems and stability, J. Math. Anal. Appl. 47 (1974), $32-42$.

7. M. J. Powers, Lefschetz fixed point theorems for a new class of multivalued maps, Pacific J. Math. 42 (1972), 211-220.

8. E. H. Spanier, Algebraic topology, McGraw-Hill, New York, 1966. MR 35 \# 1007.

9. H. Steinlein, Ein Satz über den Leray-Schauderschen Abbildungsgrad, Math. Z. 126 (1972), 176-208. MR 47 \#5667.

10. P. P. Zabreiko and M. A. Krasnosel'skiī, Iterations of operators and fixed points, Dokl. Akad. Nauk SSSR 196 (1971), 1006-1009 = Soviet Math. Dokl. 12 (1971), 294-298. MR 47 \#4082.

Institut für Angewandte Mathematik, Universität Bonn, Bonn, Federal Republic of GERMANY 